\title{
Oxidative stress activates red cell adhesion to laminin in sickle cell disease
}

Haematologica 2021

Volume 106(9):2478-2488

\section{Correspondence:}

WASSIM EL NEMER

wassim.el-nemer@efs.sante.fr

Received: June 2, 2020.

Accepted: August 12, 2020.

Pre-published: August 27, 2020.

https://doi.org/10.3324/haematol.2020.261586

(C)2021 Ferrata Storti Foundation

Material published in Haematologica is covered by copyright. All rights are reserved to the Ferrata Storti Foundation. Use of published material is allowed under the following terms and conditions:

https://creativecommons.org/licenses/by-nc/4.0/legalcode. Copies of published material are allowed for personal or internal use. Sharing published material for non-commercial purposes is subject to the following conditions:

https://creativecommons.org/licenses/by-nc/4.0/legalcode, sect. 3. Reproducing and sharing published material for commercial purposes is not allowed without permission in writing from the publisher.

\author{
Maria Alejandra Lizarralde-Iragorri, ${ }^{1,2,3 *}$ Sophie D. Lefevre, ${ }^{1,2,3^{*}}$ \\ Sylvie Cochet, ${ }^{1,2,3}$ Sara El Hoss, ${ }^{1,2,3}$ Valentine Brousse, ${ }^{1,2,3,4}$ Anne Filipe, ${ }^{1,2,3.5}$ \\ Michael Dussiot, ${ }^{6}$ Slim Azouzi, ${ }^{1,2,3}$ Caroline Le Van Kim, ${ }^{1,2,3}$ \\ Fernando Rodrigues-Lima, ${ }^{5}$ Olivier Français, ${ }^{7}$ Bruno Le Pioufle, ${ }^{8}$ Thomas Klei, ${ }^{9}$ \\ Robin van Bruggen ${ }^{9}$ and Wassim El Nemer ${ }^{1,2,3}$
}

*MALI and SDL contributed equally as co-first authors.

${ }^{1}$ Université de Paris, UMR S1134, BIGR, INSERM, Paris, France; ${ }^{2}$ Institut National de la Transfusion Sanguine, Paris, France; ${ }^{3}$ Laboratoire d'Excellence GR-Ex, Paris, France; ${ }^{4}$ Service de Pédiatrie Générale et Maladies Infectieuses, Hôpital Universitaire Necker Enfants Malades, Paris, France; ${ }^{5}$ Université de Paris, BFA, UMR 8251, CNRS, Paris, France; ${ }^{6}$ Institut Imagine, INSERM U1163, CNRS UMR8254, Université Paris Descartes, Hôpital Necker Enfants Malades, Paris, France; ${ }^{7}$ ESYCOM, Université Gustave Eiffel, CNRS UMR 9007, ESIEE Paris, Marne-la-Vallee, France; ${ }^{8}$ Université Paris-Saclay, ENS Paris-Saclay, CNRS Institut d'Alembert, LUMIN, Gif sur Yvette, France and ${ }^{9}$ Department of Blood Cell Research, Sanquin Research and Lab Services and Landsteiner Laboratory, Academic Medical Center, University of Amsterdam, Amsterdam, the Netherlands

\section{ABSTRACT}

$\mathrm{V}$ aso-occlusive crises are the hallmark of sickle cell disease (SCD). They are believed to occur in two steps, starting with adhesion of deformable low-dense red blood cells (RBC), or other blood cells such as neutrophils, to the wall of post-capillary venules, followed by trapping of denser $\mathrm{RBC}$ or leukocytes in the areas of adhesion because of reduced effective lumen-diameter. In SCD, RBC are heterogeneous in terms of density, shape, deformability and surface proteins, which accounts for the differences observed in their adhesion and resistance to shear stress. Sickle RBC exhibit abnormal adhesion to laminin mediated by $\mathrm{Lu} / \mathrm{BCAM}$ protein at their surface. This adhesion is triggered by Lu/BCAM phosphorylation in reticulocytes but such phosphorylation does not occur in mature dense RBC despite firm adhesion to laminin. In this study, we investigated the adhesive properties of sickle RBC subpopulations and addressed the molecular mechanism responsible for the increased adhesion of dense RBC to laminin in the absence of Lu/BCAM phosphorylation. We provide evidence for the implication of oxidative stress in post-translational modifications of Lu/BCAM that impact its distribution and cis-interaction with glycophorin $\mathrm{C}$ at the cell surface activating its adhesive function in sickle dense RBC.

\section{Introduction}

Sickle cell disease (SCD) is an autosomal recessive disorder caused by a single mutation in the sixth codon of the $\beta$-globin gene resulting in the expression of an abnormal hemoglobin that polymerizes under hypoxic conditions driving red blood cell (RBC) sickling. ${ }^{1}$ SCD is a multisystem disease characterized by hemolytic anemia, recurrent painful vaso-occlusive crises (VOC), stroke, acute chest syndrome, organ failure and high susceptibility to infections., ${ }^{2,3}$ On the cellular level, SCD is characterized by dehydration and RBC sickling, which decrease cell deformability and increase rigidity resulting in altered blood rheology and microcirculatory flow. ${ }^{2-6}$ In addition, RBC are known to be highly adhesive in SCD.-9 This abnormal adhesion to the endothelium is a contributing factor of the VOC and is believed to be triggered by signaling cascades that activate adhesion proteins at the red cell surface. ${ }^{10} \mathrm{~A}$ two-step model, based on in vivo vaso-occlusion observations in SCD mouse models, postulates that adhesion of deformable low-dense $\mathrm{RBC}$ and stress reticulocytes reduces effective lumen-diameter of post-capillary venules promoting selective trapping of the denser and misshapen $\mathrm{RBC}$ in the areas 
of adhesion. However, random precapillary obstruction by a small number of dense RBC also contributes to VOC as well as the entrapment of leukocytes and platelets. ${ }^{11-14}$

Sickle RBC are very heterogeneous in terms of age, shape and surface proteins. These variabilities account for the differences observed in cell adhesion and resistance to shear stress under flow conditions. ${ }^{11}$ In SCD, among other proteins and mechanisms, adhesion proteins LW/ICAM-4 (Landsteiner-Wiener/intercellular adhesion molecule-4) and Lu/BCAM (Lutheran/basal cell adhesion molecule) are abnormally activated and believed to prime adhesion of RBC to endothelial cells and/or subendothelial matrix proteins exposed to the bloodstream following vascular damage, contributing to microvasculature blockade. ${ }^{10,15-21}$

$\mathrm{Lu} / \mathrm{BCAM}$ is an adhesion molecule with wide tissue distribution. ${ }^{22,23} \mathrm{Lu} / \mathrm{BCAM}$-mediated cell adhesion to laminin can be triggered either by the phosphorylation of its serine $621^{17,24}$ or by the dissociation of its cytoplasmic domain from the spectrin-based skeleton. ${ }^{25,26}$ In SCD, phosphorylation of $\mathrm{Lu} / \mathrm{BCAM}$ was shown to occur in low-density (LD) $\mathrm{RBC}^{27}$ mainly reticulocytes, consistent with the adhesion of these cells to laminin. ${ }^{27,28}$ However, despite firm adhesion to laminin of high-density (HD) RBC, Lu/BCAM phosphorylation is very minor in this subpopulation and these cells do not respond to cAMP inducers such as forskolin. ${ }^{28}$ The mechanism underlying this increased adhesion is still unknown.

In this study, we investigated the molecular mechanism responsible for the increased adhesion of sickle HD RBC to laminin. We provide evidence for the implication of oxidative stress in post-translational modifications of $\mathrm{Lu} / \mathrm{BCAM}$ that impact its distribution and cis-interactions at the cell surface and activate its adhesive function.

\section{Methods}

\section{Patients}

The study was conducted in accordance with the Declaration of Helsinki and was approved by the Regional Ethics Committee (n`3215 CPP Ile de France III). Blood samples were recovered from blood tubes drawn for medical care at Necker Hospital (Paris) after written informed consent. Blood samples were collected on ethylenediaminetetraacetic acid (EDTA) from a total of 39 patients with sickle cell anemia (SS and $\mathrm{S} \beta^{\circ}$ genotypes) (females and males; median age: 8 years [Min-Max, 2-53 years]), and from 26 healthy donors (age range, 18-70, as per Etablissement Français du Sang [EFS] criteria). Sickle patients were not on a regular transfusion program nor under hydroxyurea $(\mathrm{HU})$ treatment. All experiments were performed with fresh blood samples, within 2 hours after blood was drawn.

\section{Percoll fractionation}

RBC subpopulations were obtained from sickle whole blood fractionation as previously described, ${ }^{27}$ using a Percoll triple-density fractionation (densities: 1.076, 1.096, and 1.11). Three different density layers were obtained and collected as follows: LD (low density, rich in reticulocytes), D (dense), and HD (high density, rich in irreversibly sickled cells).

\section{Microfluidic assays}

The microfluidic filtering design is based on eight mechanical filtering units associated in parallel and connected together with a microchannel network. Each filtering unit is composed of five parallel rows comprising pillars of $15 \mu \mathrm{m}$ diameter separated by slits of 10, 8, 7, 6 or $5 \mu \mathrm{m}$ (Figure 1A) or four parallel rows with slits of 5, 4, 3 or 2 um (Online Supplementary Figure S1). Side flow is rendered possible in the device, the $\mathrm{U}$ form filter zone comprises pillars with a $5 \mu \mathrm{m}$ gap between them. In order to reduce the hydraulic resistance of the full design, the microchannel network is $25 \mu \mathrm{m}$-high compared to the $5 \mu \mathrm{m}$ height of each filtering unit (Figure 1A). The microfluidic device was made of polydimethylsiloxane (PDMS, Sylgard), a silicone elastomer, ${ }^{29}$ using standard microfabrication and molding. The mold was fabricated by the micro-patterning of two successive SU8 photoresist layers (Microchem, Newton, MA) to obtain a two-levels negative mold on a 4-inch Silicon substrate. The SU8 layers thicknesses were $5 \mu \mathrm{m}$ and $25 \mu \mathrm{m}$, corresponding respectively to the height of the filtering units and the microchannels network. A mixture of PDMS and curing agent was poured on the SU8 mold, and reticulated at $75^{\circ} \mathrm{C}$ for 2 hours. Access through-holes were then punched, using biopsy punchers (diameter of $1.5 \mathrm{~mm}$ ). The PDMS device, with open channels formed on one of its sides, was then assembled to a microscope coverslip, using $\mathrm{O}_{2}$ plasma activation $(30 \mathrm{~W}, 300 \mathrm{mT}, 20 \mathrm{~s}$ ) to achieve a covalent bonding. Luer (TM) connectors were then inserted at the inlet and outlet of this microfluidic device, to achieve the sample injection with the flow controller.

For each assay, $10 \mu \mathrm{L}$ of RBC pellet were stained with either PKH67 fluorescent Cell Linker Kit (green) or PKH26 fluorescent Cell Linker Kit (red) according to the manufacturer's instructions (Sigma Aldrich). A 1\% hematocrit solution in CellStab containing equal concentration of green and red stained RBC were loaded in the input well of the chip and perfused at constant pressure (250 mBar) using an MFCS ${ }^{\mathrm{TM}}-\mathrm{EZ}-1 \mathrm{C}$ pump (Fluigent). RBC trapping within each filtering unit was monitored over time by sequential fluorescence images acquired using an inverted AxioObserver Z1 microscope coupled with a high resolution AxioCam MRm Rev. 3 camera (Carl Zeiss). Green and red fluorescent RBC were visualized using the 470 and $555 \mathrm{~nm}$ Colibri LED (Carl Zeiss), respectively. Images were then analyzed using ImageJ software. ${ }^{30}$

\section{Flow adhesion assays and red blood cell counting}

$\mathrm{RBC}$ adhesion to Laminin 521 was evaluated under flow conditions using capillary flow chambers. Recombinant human Laminin 521 (BioLamina, Sundbyberg, Sweden) at $5 \mathrm{ng} / \mu \mathrm{L}$ was immobilized in Vena8 Endothelial+TM biochips (internal channel dimensions: length $20 \mathrm{~mm}$, width $0.8 \mathrm{~mm}$, height $0.12 \mathrm{~mm}$ ). RBC were perfused at $5.10^{7} \mathrm{RBC} / \mathrm{mL}$ for $10 \mathrm{~min}$ at $0.5 \mathrm{dyn} / \mathrm{cm}^{2}$ and 6 min washouts were performed at 1, 2, 3, 4, 5, 6, 7, 8, 9 and 10 $\mathrm{dyn} / \mathrm{cm}^{2}$ using the ExiGo ${ }^{\mathrm{TM}}$ pump (Cellix Ltd, Dublin, Ireland). After each wash, images of adherent $\mathrm{RBC}$ were taken using the AxioObserver Z1 microscope (10x objective) (Carl Zeiss, Le Pecq, France). Adherent RBC were counted on each field using Image J.

The number of immobile RBC was assessed by using the Image Calculator option of the Image J software. ${ }^{30}$ The picture of one area at $2 \mathrm{dyn} / \mathrm{cm}^{2}$ was combined to the picture of the same area taken at $3 \mathrm{dyn} / \mathrm{cm}^{2}$. On the newly created image, immobile cells appeared in dark grey whereas cells present on only one of the two combined images appeared in light grey. Dark grey objects were counted with Image J software after setting an appropriate threshold.

\section{Control blood sample oxidation}

Control RBC were washed with phospate buffered saline (PBS) 1X (Thermo Fisher), suspended at $20 \%$ hematocrit in either PBS or $270 \mu \mathrm{M}$ cumene hydroperoxide (SIGMA-ALDRICH) and incubated for 2 hours with constant mild shaking. After incubation, RBC suspensions were centrifuged, the supernatant was discarded and the RBC pellets were used for subsequent experiments. 


\section{Confocal microscopy}

Imaging was performed on the Confocal LSM 510 META-TIRF (Zeiss, Oberkochen, Germany). LASX software was used to set up and analyze the experiments (Leica microsystems, Wetzlar, Germany).

\section{Imaging flow cytometry assays}

Expression of Lu/BCAM on the RBC surface was analyzed using F241 mouse monoclonal antibody. After 1-hour incubation with F241 (dilution [d]: 1/10), the secondary anti-mouse APC-conjugated antibody (d: 1/100) (Beckman Coulter) was added for 1 hour, then RBC were washed and suspended in $200 \mu \mathrm{L}$ of thiazole orange (TO) dye (Retic-CountTM, Becton-Dickinson) for $30 \mathrm{~min}$ utes (min) to label reticulocytes. RBC were analyzed using ImageStream®X Mark II Imaging Flow Cytometer (Merck Millipore) (60x magnification) and the IDEAS software (version 6.2). Lu/BCAM-positive mature RBC (Lu APC) were gated, excluding the reticulocytes ( $\mathrm{TO}$-positive events). Using the

A

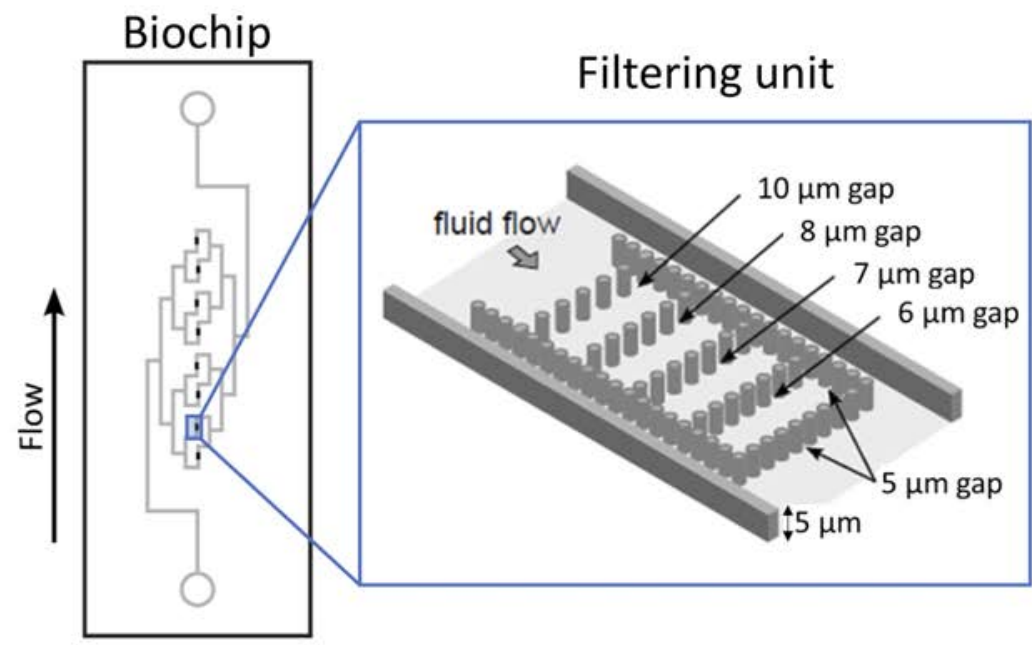

B

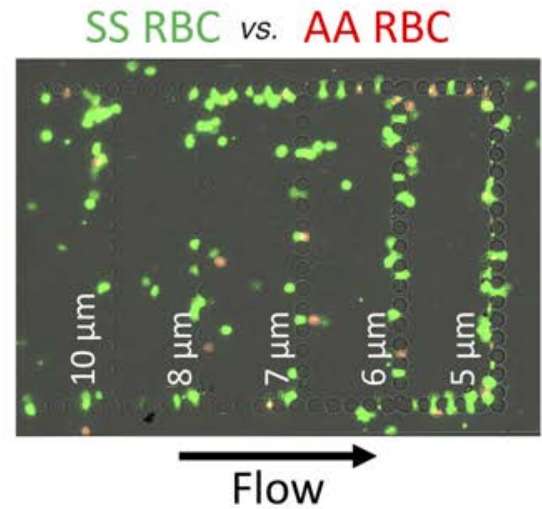

D

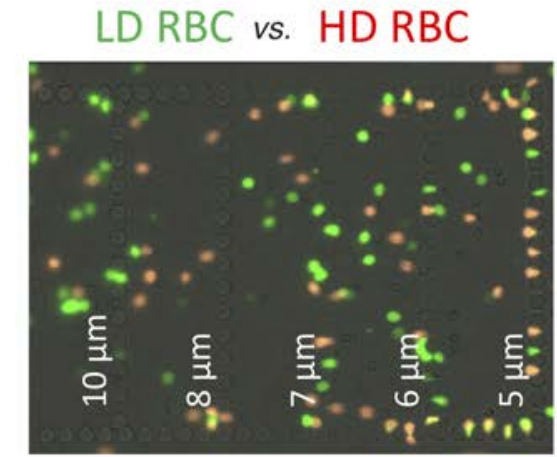

C

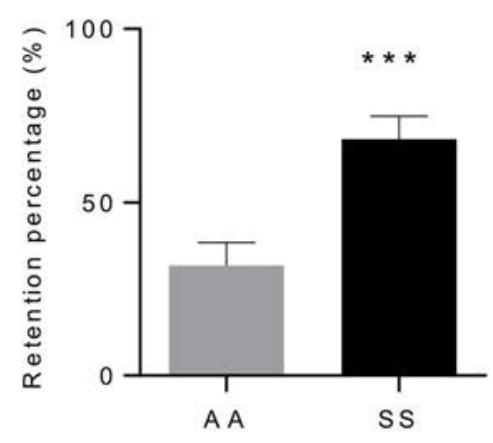

$\mathbf{E}$

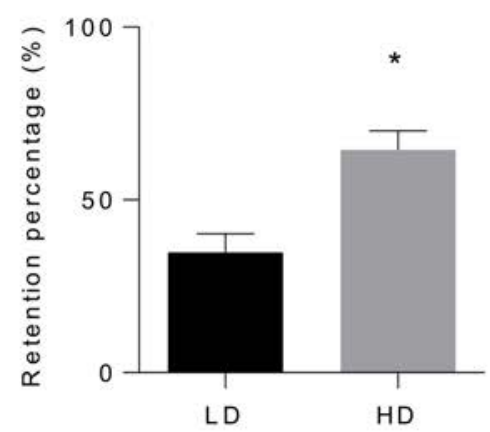

Figure 1. Analysis of red blood cell deformability using a microfluidic biomimetic chip. (A) Left panel: the microfluidic device comprises eight filtering units arranged in parallel. Right panel: each filtering unit is $5 \mu \mathrm{m}$-high and has a $U$ shape composed of a series of $15 \mu \mathrm{m}$ pillars separated by $5 \mu \mathrm{m}$ slits, with two 10 um-wide side channels. Inside the $U$ shape, four rows are disposed in parallel with decreasing slit width $(10,8,7$ and 6 um). (B) Microscopy image showing SS red blood cells (RBC) (green) and AA RBC (red) trapped into the filtering unit slits. (C) Retention percentage of AA and SS RBC in the $5 \mu \mathrm{m}$ slits. Mann-Whitney test, $* \star \star P<0.0001$ (D) Microscopy image showing low-density (LD) RBC (green) and high-density (HD) RBC (red) trapped into the peripheral $5 \mu \mathrm{m}$ slits of the filtering unit. The majority of the other cells are in motion in the space separating two consecutive walls (E) Retention percentage of LD and HD RBC in the peripheral 5 um slits. Wilcoxon test, $* P<0.05$. In the graphs $\mathrm{C}$ and $\mathrm{E}$ the data is expressed as the percentage of cells from each RBC type trapped into the $5 \mu \mathrm{m}$ slits, considering the total number of cells trapped into the $5 \mu \mathrm{m}$ slits as $100 \%(n=7)$. 
A

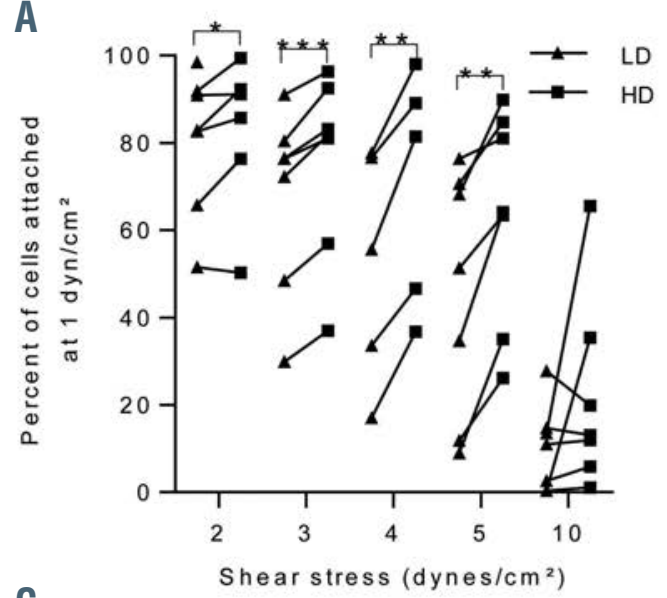

C

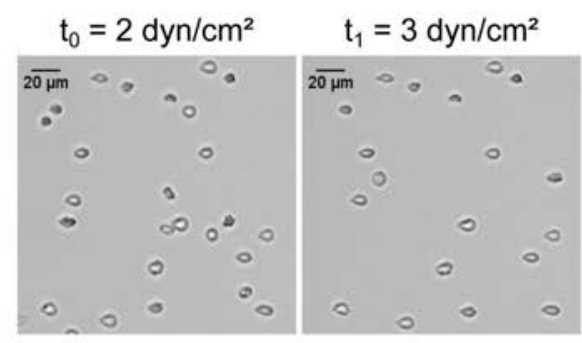

immobile cells between 2 and 3 dyn $/ \mathrm{cm}^{2}$

E

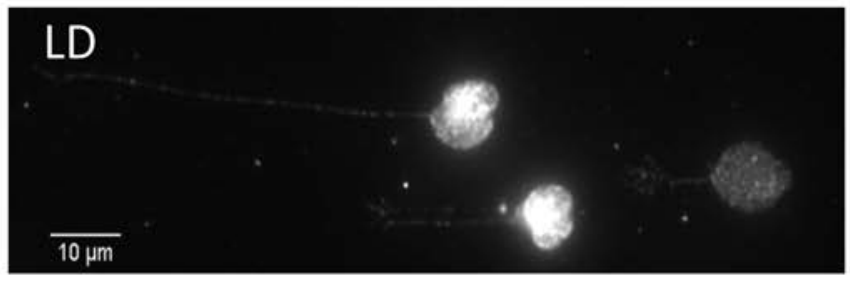

B

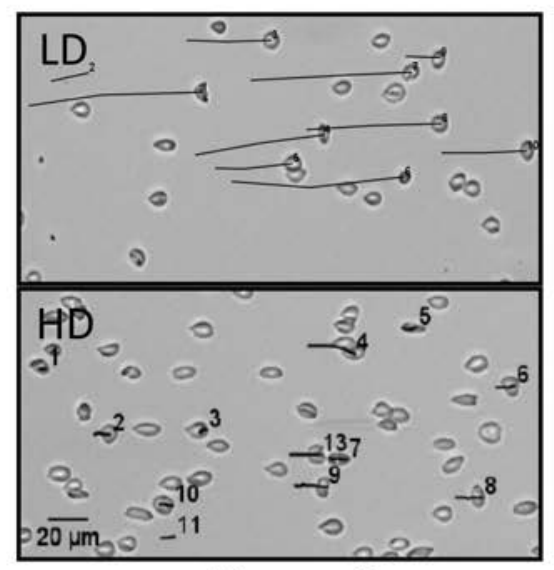

Flow $\rightarrow$

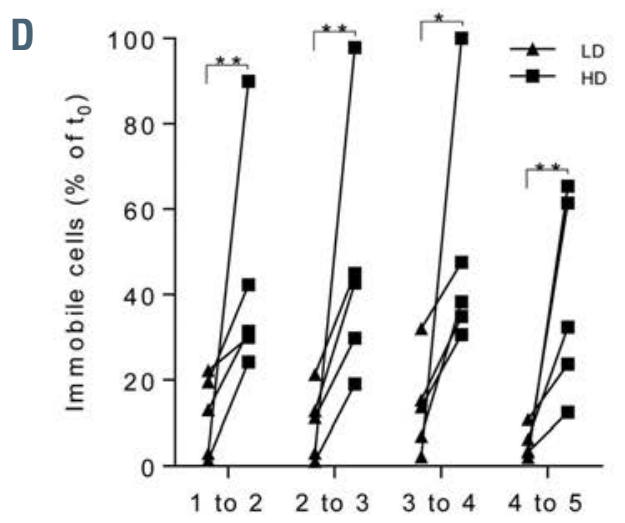

Shear stress $\left(\right.$ dyn $\left./ \mathrm{cm}^{2}\right)$
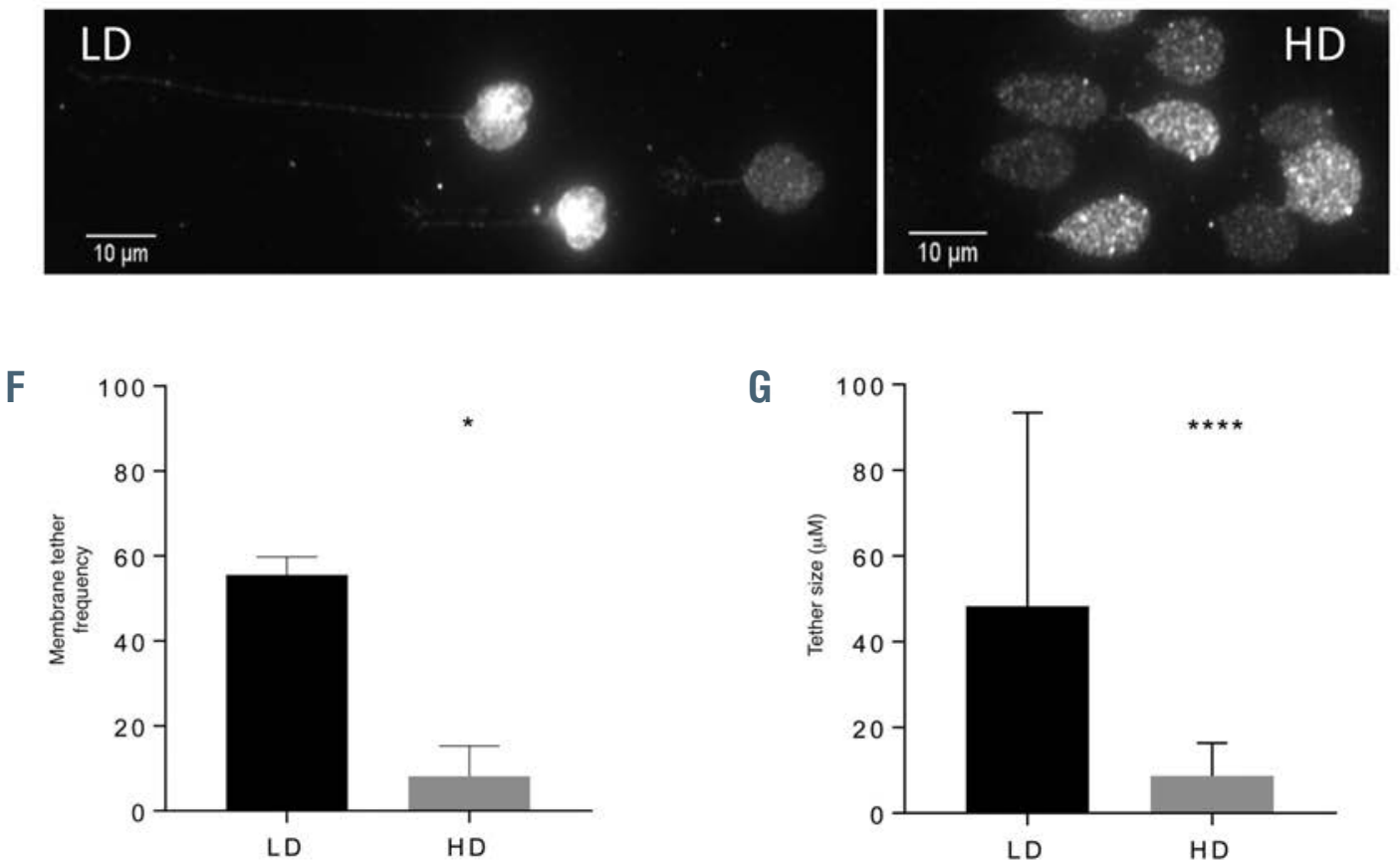
Figure 2. Adhesion to laminin and shear stress resistance of SS red blood cell subpopulations. (A). Adhesion assays under flow conditions were done by perfusing low-density (LD) and high-density (HD) red blood cells (RBC) from seven sickle cell disease (SCD) patients through a biochip coated with Laminin 521. The amount of RBC, of each subpopulation and each blood sample, attached at $1 \mathrm{dyn} / \mathrm{cm}^{2}$ was considered as $100 \%$ and used to determine the percentage of attached RBC at increasing shear stress, as indicated on the x-axis. The percentage of HD RBC $(\mathbf{\square})$ attached to laminin was higher than the percentage of LD RBC ( $\mathbf{\Delta})$. Paired $t$-test, $\star \star P<0.005$. (B) Tracking of mobile cells during a flow adhesion assay for LD (upper panel) and HD (lower panel) RBC at 3 dyn/cm². (C) Computational treatment of brightfield images of the same field to identify immobile cells between 2 and $3 \mathrm{dyn} / \mathrm{cm}^{2}$ during a flow adhesion assay. Overlaid image (bottom panel) revealing immobile cells in dark grey and mobile cells in light grey (D) Quantification of immobile cells for LD ( $\mathbf{\Delta}$ ) and HD ( $\mathbf{m})$ RBC from blood samples of four SCD patients. Results are expressed as percentage of immobile cells between two consecutive shear stress values. Paired t-test, *P<0.05. (E) Representative images of Lu/BCAM detected by immunofluorescence as well as tether length of LD and HD RBC in a flow adhesion assay at high shear stress (7 dyn/cm²). (F) Percentage of tether-containing cells in each RBC subpopulation at $7 \mathrm{dyn} / \mathrm{cm}^{2}$. Paired $t$-test, $* P<0.05$. (G) Tether length in LD and HD RBC. Mann-Whitney test, $* * * * P<0.0001$.

Modulation Feature, that measures the intensity range of an image, normalized between 0 and 1 (formula: Modulation $=$ Max Pixel - Min Pixel / Max Pixel + Min Pixel), reflecting the fluorescent signal distribution, we defined two subpopulations of mature Lu/BCAM RBC: Low-Modulation (Spots) and High-Modulation (Patches). Based on the $x$-axis Modulation_M11_Ch11 APC and y-axis Mean Pixel_M11_Ch11 APC, the "Spots" population was between -0.039 and 0.231 , and the "Patches" population was between 0.235 and 0.552 .

\section{Flow cytometry assays}

Protein sialylation was measured by incubating RBC suspensions with biotinylated lectin $(35 \mathrm{ng} / \mathrm{mL}$ ) (Maackia amurens Lectin II, VECTOR) for 1 hour with constant shaking. After washes, Streptavidin-488 $(10 \mu \mathrm{g} / \mathrm{mL}$ ) (Streptavidin Alexa Fluor 488 conjugate, Invitrogen) was added to the pellet, and incubated for $30 \mathrm{~min}$ in the dark. Glycophorin-C (GPC) sialylation on mature RBC was determined by incubating the RBC suspensions with the antiBRIC 4 (dilution [d]: 1/100) antibody or anti-BRIC 10 (d: 1/200) antibody (IBGRL Research Products). After 1-hour incubation at room temperature and several washes, the RBC pellet was incubated with the secondary anti-mouse APC antibody (d: 1/100) (Beckman Coulter) for $45 \mathrm{~min}$ in the dark. RBC were analyzed using a BD FACS Canto II (BD Biosciences), the data obtained was analyzed using the FCS Express 6 software (De Novo).

\section{Statistical analyses}

Data was analyzed by two-tailed Mann-Whitney or Wilcoxon test, and Paired $t$-test using the GraphPad Prism 7.00 software. ${ }^{*} P \leq 0.05,{ }^{* *} P \leq 0.01,{ }^{* *} P \leq 0.001$ and ${ }^{* * * *} P \leq 0.0001$ were considered significant.

\section{Results}

\section{Validating high-density and low-density red blood cell isolation Deformability of AA and SS red blood cells}

$\mathrm{HD} \mathrm{RBC}$ are known to be rigid cells with reduced deformability, which contributes to capillary blockade in vivo. In order to validate the fractionation method used to isolate $\mathrm{LD}$ and $\mathrm{HD}$ RBC from SCD blood samples, we assessed their deformability after isolation at the single cell level using a microfluidic approach based on perfusing $\mathrm{RBC}$ in a spleen-like biomimetic chip with filtering units comprising slits from 5 down to $2 \mu \mathrm{m}^{31}$ (Online Supplementary Figure S1). First, we analyzed the deformability of total RBC using this biochip. AA and SS RBC were fluorescently labeled with PKH26 (red) and PKH67 (green), respectively, and mixed at a $1: 1$ ratio into a suspension at $0.1 \%$ hematocrit. Perfusing this RBC suspension led to a total blockade of the biochip indicating that the slit dimensions were not suitable for testing deformability of SS RBC. As the blockade occurred at the $5 \mu \mathrm{m}$ wall we designed a new biochip with slit dimensions of 10 to $5 \mu \mathrm{m}$ (Figure 1A). Perfusing the 1:1 AA-SS RBC suspen- sion into this biochip showed preferential trapping of SS RBC (Figure 1B), with these cells showing a higher retention rate than $A A R B$ (Figure $1 C$ ), indicating that the biochip was a good tool to assess RBC deformability in the SCD context.

\section{Deformability of low-density and high-density sickle red blood cells}

RBC suspensions were prepared with PKH67-labeled LD RBC and PKH26-labeled HD RBC (1:1) and perfused in the biochip. Both LD and HD RBC were retained in the biochip, mainly at the $5 \mu \mathrm{m}$ wall, indicating the presence of rigid cells in both populations (Figure 1D). Quantification of both $\mathrm{RBC}$ types retained in the $5 \mu \mathrm{m}$ peripheral slits showed more HD than LD RBC (Figure 1E) indicating that $\mathrm{RBC}$ from the $\mathrm{HD}$ fraction were less deformable than those from the LD fraction, thus validating our fractionation method based on cell density.

\section{High-density red blood cells are more resistant to shear stress and adhere more firmly to laminin than low-density red blood cells \\ Red blood cell adhesion to laminin under flow conditions}

We assessed the adhesive properties and resistance to shear stress of RBC sub-populations by performing adhesion assays under flow conditions with LD and HD RBC from seven SCD patients using channels coated with Laminin 521. Both RBC types showed significant adhesion to laminin but there was a difference between them regarding resistance to shear stress. This resistance was first assessed by calculating the percentage of cells adhering at a given shear stress considering the number of adhering cells at $1 \mathrm{dyn} / \mathrm{cm}^{2}$ as $100 \%$. HD RBC were more resistant than LD RBC at physiological shear stresses for capillaries or post-capillary venules $\left(2-5 \mathrm{dyn} / \mathrm{cm}^{2}\right)$, while at high shear stress $\left(10 \mathrm{dyn} / \mathrm{cm}^{2}\right)$ both cell types showed no significant difference (Figure 2A). Exploring the cellular dynamics, we observed that LD RBC comprised a higher proportion of rolling cells than $\mathrm{HD} \mathrm{RBC}$ (Online Supplementary Video S1), with a higher proportion of mobile cells covering a greater distance within the same time frame (Figure 2B). We assessed cell dynamics by determining the percentage of immobile cells between two consecutive shear stress steps. In order to do so, computational treatment of brightfield images of the same fields at two consecutive shear stresses was done to identify and count the cells that remained at the same spot between the two time-points (Figure 2C; Online Supplementary Figure S2A). The percentage of immobile cells was constantly higher for HD than LD RBC (Figure 2D), indicating that HD RBC were more firmly attached to laminin than LD RBC even at high shear stress.
Lu/BCAM expression pattern on adherent low-density and high-density red blood cells


A
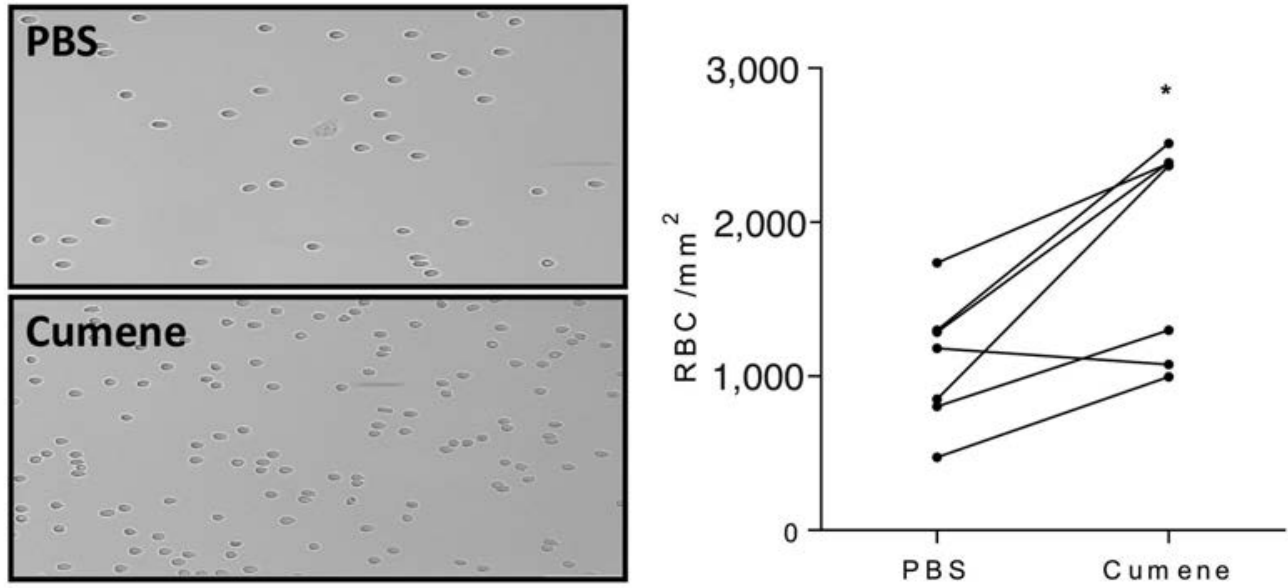

B
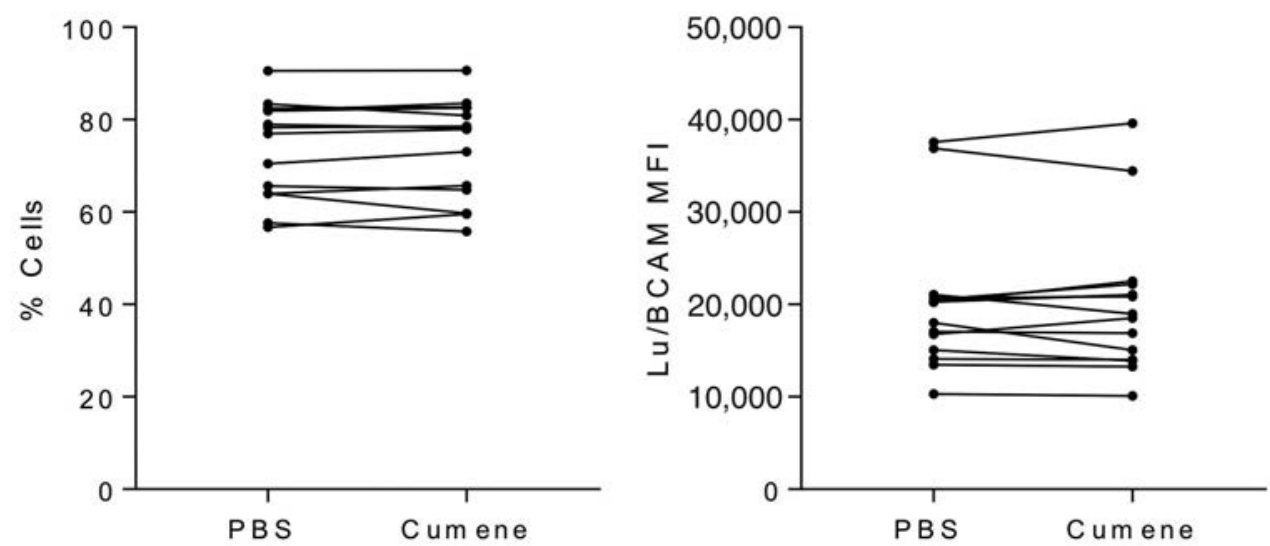

Figure 3. Effect of in vitro oxidation on AA red blood cell adhesion to laminin. (A) Left panel: typical microscopy images of non-oxidized and cumene hydroperoxideoxidized AA red blood cells (RBC) adhering to Laminin 521 at $3 \mathrm{dyn} / \mathrm{cm}^{2}$. Right panel: quantification of cell adhesion showing the mean number of adherent RBC/mm ${ }^{2}$ in seven oxidized and non-oxidized AA RBC samples at $3 \mathrm{dyn} / \mathrm{cm}^{2}$. Wilcoxon test, $* P<0.05$. (B) Flow cytometry analysis of Lu/BCAM expression at the RBC surface expressed as percentage of Lu/BCAM-positive RBC (left panel) and mean fluorescence intensity (MFI) of these RBC (right panel) under non-oxidized (phosphate buffered saline $[\mathrm{PBS}]$ ) and oxidized conditions (cumene). No significant differences were observed, Wilcoxon test, $P=0.0714$.

In order to gain insight into the potential mechanism underlying this difference between LD and HD RBC, cells were fixed after the $7 \mathrm{dyn} / \mathrm{cm}^{2}$ step, stained fluorescently for Lu/BCAM and analyzed by confocal microscopy. There was a difference in the expression pattern of $\mathrm{Lu} / \mathrm{BCAM}$ between $\mathrm{LD}$ and $\mathrm{HD} \mathrm{RBC}$ at the interface with laminin. HD RBC showed a homogeneous distribution of $\mathrm{Lu} / \mathrm{BCAM}$ with some cells showing intense staining and the presence of bigger spots suggestive of potential Lu/BCAM aggregates (Figure 2E; Online Supplementary Figure S2B). LD RBC showed cells with a smaller surface contact area, large fluorescent patches and very fine fluorescent membrane extensions tethering the cells to the surface (Figure 2E). The proportion of RBC exhibiting membrane tethers was higher in LD $(55.6 \%)$ than in HD $\mathrm{RBC}(8.2 \%)$ (Figure $2 \mathrm{~F})$, and tethers were also longer in $\mathrm{LD}$ RBC (Figure $2 \mathrm{G}$ ) suggesting a more dynamic lipid bilayer in LD RBC.

\section{Oxidation activates AA red blood cell adhesion to laminin}

We have previously shown that Lu/BCAM-mediated adhesion to laminin is activated by phosphorylation of serine 621 of its cytoplasmic tail. ${ }^{17}$ In SCD, this phosphorylation takes place in reticulocytes, with very low levels of phosphorylation detected in $\mathrm{HD} \mathrm{RBC}^{27}$ Considering the high levels of adhesion of HD RBC, we hypothesized that Lu/BCAM might be activated by post-translational modifications triggered by oxidative stress. In order to test this hypothesis, we assessed adhesion of control (AA) $\mathrm{RBC}$ under oxidative conditions after incubation with cumene hydroperoxide $(270 \mu \mathrm{M})$, an agent that induces membrane lipid peroxidation. AA RBC showed the expected residual adhesion to laminin, that was significantly increased after incubation with cumene hydroperoxide (Figure 3A; Online Supplementary Figure S3). This was not due to a difference in the Lu/BCAM expression level between oxidative and control conditions as determined by flow cytometry measuring the percentage of Lu/BCAM-positive RBC and their mean fluorescence intensity (MFI) (Figure 3B). Moreover, this increased adhesion was not due to increased phosphorylation of Lu/BCAM as determined by western blot using an antiphosphoSerine antibody (not shown).

\section{Oxidative stress alters Lu/BCAM distribution at the red blood cell surface \\ Analysis of Lu/BCAM membrane distribution by confocal microscopy \\ We evaluated the impact of oxidation on the distribu-}


A

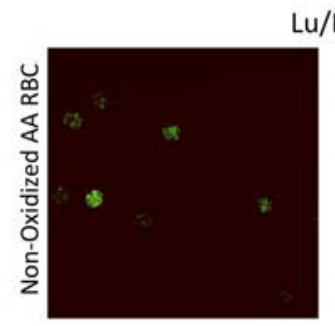

C

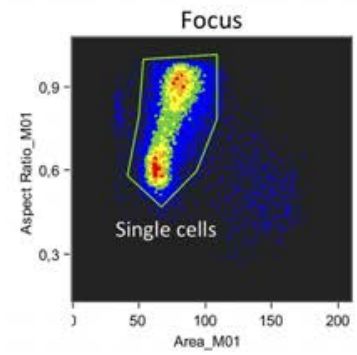

D

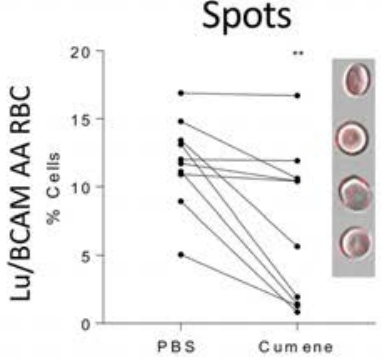

F

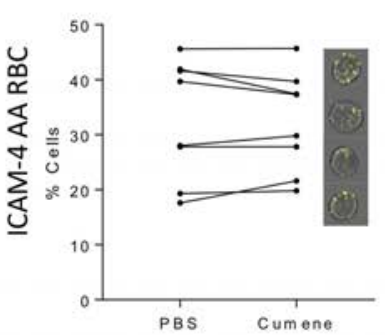

H

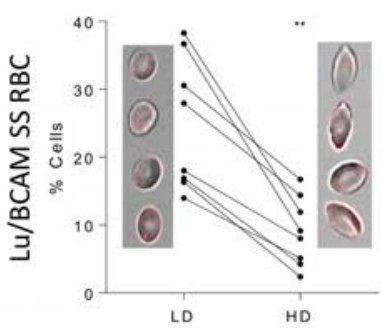

Lu/BCAM
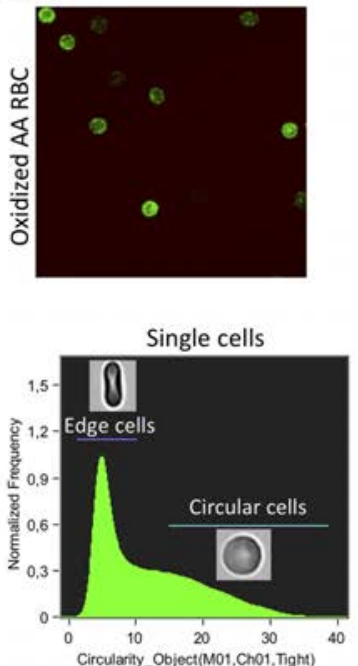

Patches
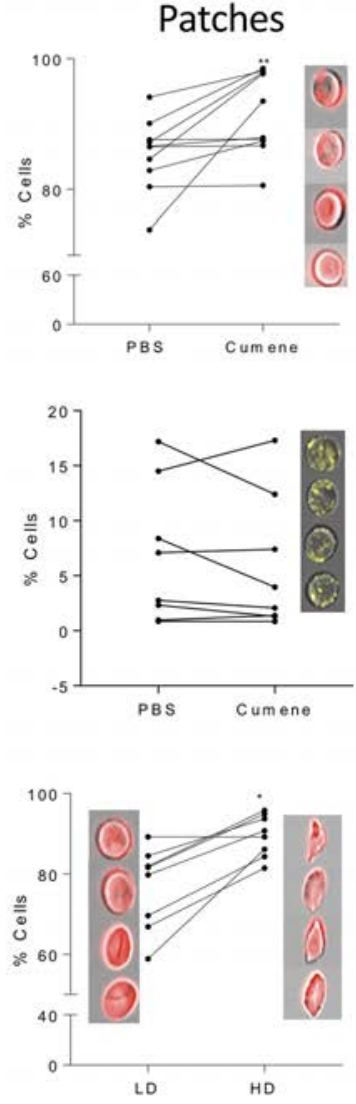

B

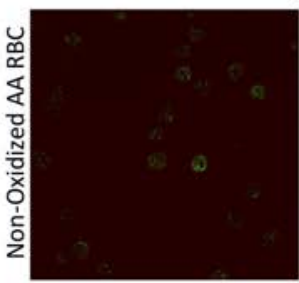

ICAM-4
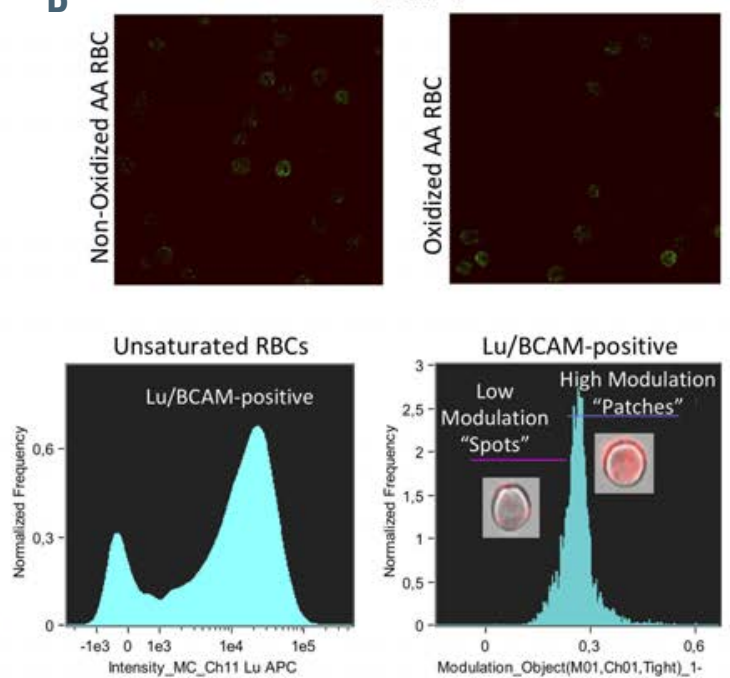

$\mathbf{E}$

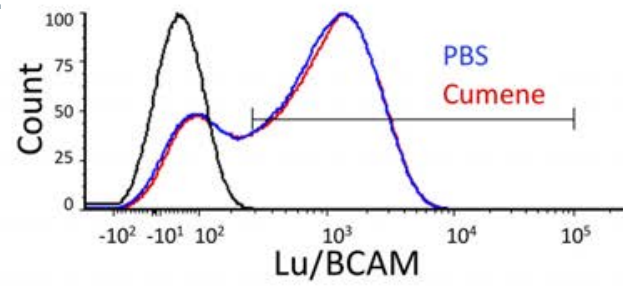

G

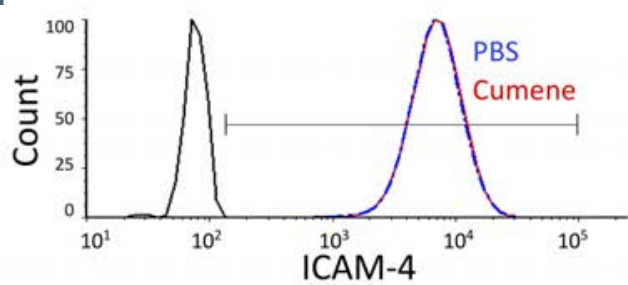

I

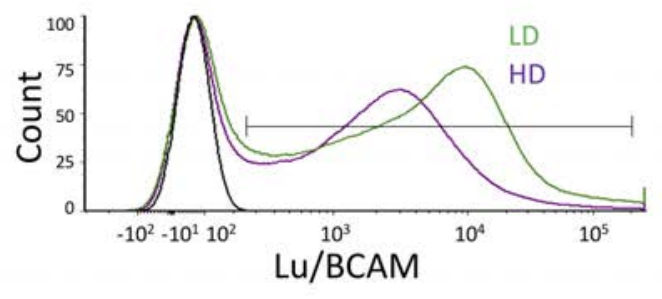

Figure 4. Expression pattern of Lu/BCAM and ICAM-4 on red blood cells by confocal microscopy and imaging flow cytometry. Confocal microscopy images of red blood cells (RBC) expressing (A) Lu/BCAM and (B) ICAM-4 under non-oxidized and oxidized conditions. (C) Imaging flow cytometry analysis steps of Lu/BCAM expression on the RBC surface. After gating the single cells, gating is set on RBC facing the camera (circular cells), then on those expressing Lu/BCAM. In the final step a modulation feature is applied to measure the intensity range and distribution of Lu/BCAM, and define two main patterns: Low Modulation (Spots) and High Modulation (Patches). (D) Percentage of Lu/BCAM-positive RBC with Spots pattern (left) and Patches pattern (right) ( $\mathrm{n}=10$ ). Wilcoxon test, $* \star P<0.01$. ( $E$ ) Representative flow cytometry plots of Lu/BCAM expression in a non-oxidized (phosphate buffered saline [PBS]) and oxidized (cumene hydroperoxide) AA RBC sample. (F) Percentage of ICAM-4-positive RBC with Spots pattern (left) and Patches pattern (right) ( $n=8$ ). Wilcoxon test, $P=0.8125$ and $P=0.3828$. (G) Representative flow cytometry plots of ICAM-4 expression in a non-oxidized (PBS) and oxidized (cumene hydroperoxide) AA RBC sample. (H) Percentage of Lu/BCAM-positive RBC with Spots pattern (left) and Patches pattern (right) in low-density (LD) and high-density (HD) SS RBC ( $=8$ ); Wilcoxon test, ** $P<0.01$, * $P<0.05$. (I) Representative flow cytometry plots of Lu/BCAM expression in LD and HD RBC from one SS blood sample. 

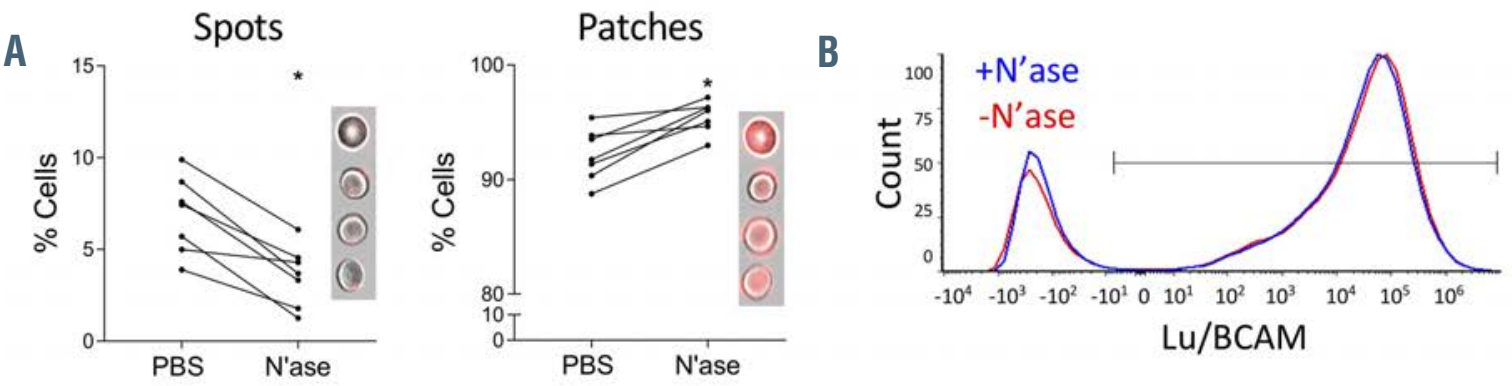

C
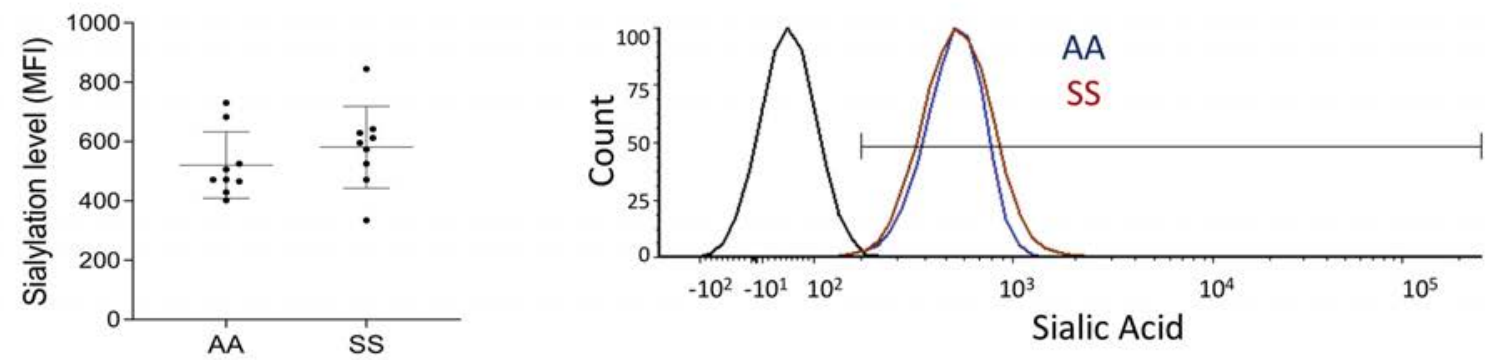

D
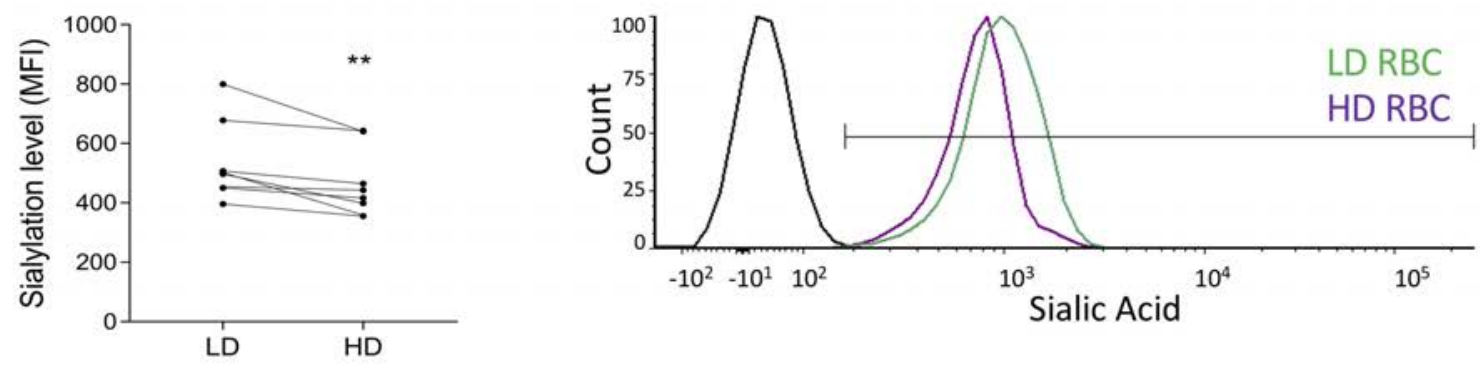

E
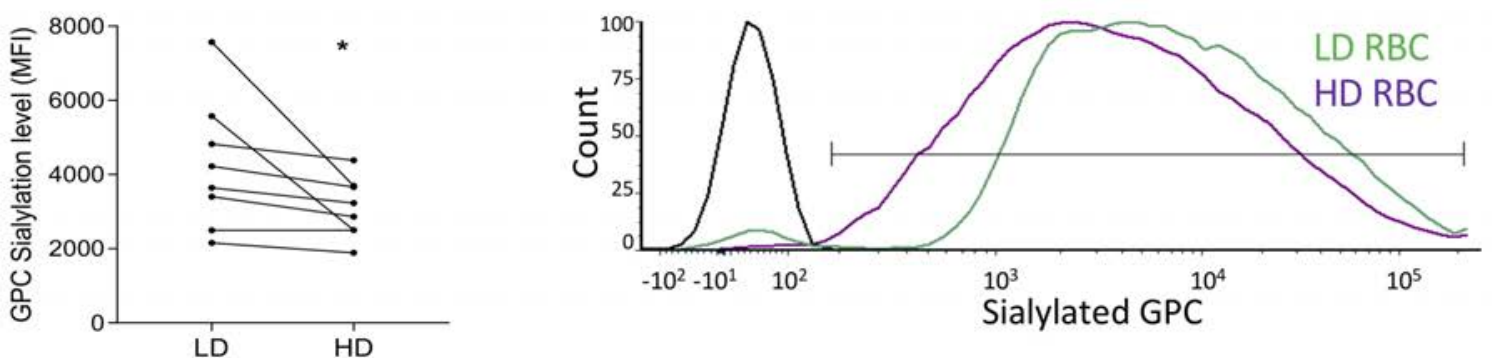

F
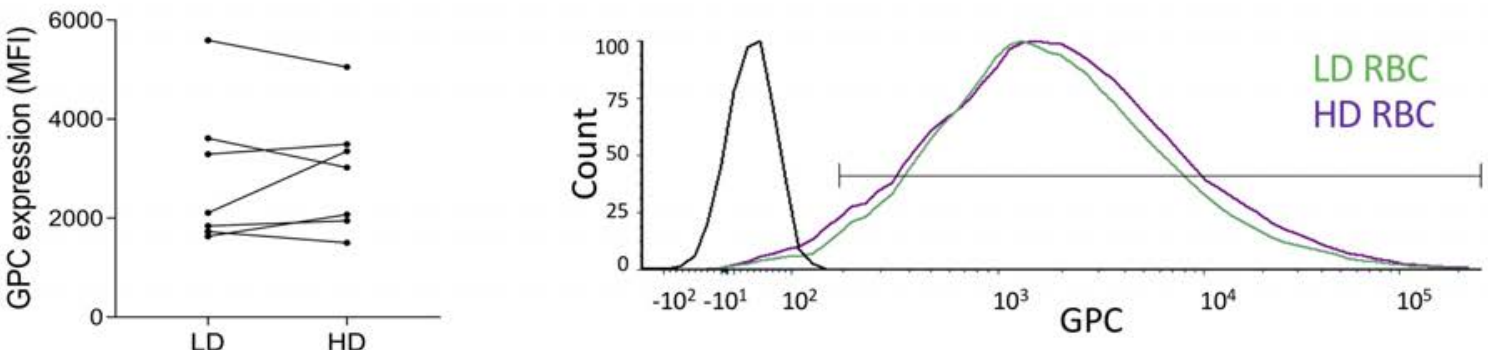

Figure 5. Sialic acid levels, glycophorin C sialylation and Lu/BCAM distribution in AA and SS red blood cells. (A) Impact of neuraminidase (N'ase) treatment on Lu/BCAM Spots and Patches patterns on AA red blood cells (RBC). Wilcoxon test $* P<0.05$. (B) Representative flow cytometry plots of Lu/BCAM expression in RBC treated or not with neuraminidase. (C) Left panel: sialic acid levels expressed as mean fluorescence intensity (MFI) on the RBC surface of 11 SS and nine AA samples. No significant difference was found between both groups. Mann-Whitney test, $P=0.224$. Right panel: representative flow cytometry plots of sialic acid distribution on one AA and one SS RBC samples. (D) Left panel: sialic acid levels expressed as MFI on the RBC surface of eight low-density (LD) and high-density (HD) samples. Wilcoxon test, $* * P<0$.01. Right panel: representative flow cytometry plots of sialic acid distribution on LD and HD RBC from the same SS blood sample. (E) Left panel: glycophorin C (GPC) sialylation levels expressed as mean fluorescence intensity (MFI) on the RBC surface of eight LD and HD samples. Wilcoxon test, $* P<0.05$. Right panel: representative flow cytometry plots of silalylated GPC on LD and HD RBC from the same SS blood sample. (F) Left panel: GPC expression represented as MFI on the RBC surface of eight LD and HD samples. No significant differences were observed. Wilcoxon test, $P=0.64$. Right panel: representative flow cytometry plots of GPC expression on LD and HD RBC from the same SS blood sample. 
tion of Lu/BCAM at the red blood cell surface using confocal microscopy. As expected and previously shown, ${ }^{26}$ $\mathrm{Lu} / \mathrm{BCAM}$ showed a punctuated expression pattern at the red blood cell surface (Figure 4A left panel; Online Supplementary Figure S4). Under oxidative conditions, there was more RBC with large fluorescent patches suggestive of Lu/BCAM aggregation (Figure 4A right panel; Online Supplementary Figure S4). In order to check if this was a general feature of membrane proteins under oxidative conditions we stained for ICAM-4, another member of the immunoglobulin superfamily, and found no difference in its membrane distribution between both conditions (Figure 4B) indicating that oxidation targets $\mathrm{Lu} / \mathrm{BCAM}$ membrane distribution through a specific mechanism.

\section{Analysis of LU/BCAM membrane distribution by imaging flow cytometry}

We assessed Lu/BCAM membrane distribution using a high throughput approach based on imaging flow cytometry. RBC were classified based on the expression pattern of $\mathrm{Lu} / \mathrm{BCAM}$ using the modulation feature that measures the intensity range and distribution of a fluorescent signal (see methods) (Figure 4C). Two main patterns were distinguished in this analysis: the "Low modulation" for weak intensity dispersed spots (Spots) and "High modulation" for strong intensity big patches (Patches) (Figure 4C, last panel). Both patterns were concomitantly present in all blood samples and all conditions. We compared the proportion of each pattern under oxidative and control conditions and found less RBC with Spots and more with Patches in the presence of cumene hydroperoxide (Figure 4D; Online Supplementary Figure S5A), with no difference in $\mathrm{Lu} / \mathrm{BCAM}$ global expression (Figure $4 \mathrm{E}$ ), indicating that oxidation induces the formation of Lu/BCAM aggregates at the cell surface. As expected, based on the confocal microscopy results, oxidation did not impact the proportions of ICAM-4 Spots and Patches subpopulations (Figure 4F; Online Supplementary Figure S5A) or its global expression at the RBC surface (Figure 4G).

Finally, we assessed Lu/BCAM distribution on SS LD and HD RBC in which both the Spots and the Patches patterns were found. Similar to oxidized AA RBC, the percentage of cells with the Patches pattern was higher in $\mathrm{HD}$ RBC in comparison with LD RBC, while the Spots pattern prevailed in the LD subpopulation (Figure 4H; Online Supplementary Figure S5B). As expected and already reported, $\mathrm{Lu} / \mathrm{BCAM}$ expression was lower in $\mathrm{HD}$ than in $\mathrm{LD}$ RBC (Figure 4I).

\section{Less glycophorin C sialylation on SS red blood cells Impact of sialic acid removal on Lu/BCAM membrane distribution \\ We have recently shown that Lu/BCAM can establish cis-interactions with GPC sialic acids at the RBC surface keeping it in a "locked" conformation impeding its interac- tion with laminin. ${ }^{32}$ In order to test if such interactions modulate the Lu/BCAM expression pattern, AA RBC were treated with neuraminidase (N'ase) in order to elim- inate protein sialic acids, labeled with an anti-Lu/BCAM antibody and analyzed by imaging flow cytometry. We found that loss of sialic acids resulted in less Spots and more Patches RBC (Figure 5A) with no impact on $\mathrm{Lu} / \mathrm{BCAM}$ global expression level (Figure $5 \mathrm{~B}$ ), indicating that lateral interactions of Lu/BCAM with sialic acid residues impede its capacity to aggregate at the cell sur-}

face. Altogether, these results suggest that oxidation may trigger Lu/BCAM release from sialic acid lateral interactions leading to its aggregation and activated adhesive function.

\section{Total sialylation and specific glycophorin C sialylation levels}

As HD RBC showed higher percentages of cells with the Patches pattern and that treatment of AA RBC with N'ase increases also the proportions of this sub-population, we hypothesized that increased HD RBC adhesion to laminin might result from aggregation of Lu/BCAM molecules subsequent to altered GPC sialylation. Using biotinylated lectins, we measured the sialic acid levels at the surface of RBC from nine AA and nine SS blood samples by flow cytometry and found no significant difference between the two groups (Figure 5C). We performed the same analysis on seven SCD fractionated blood samples and found less sialic acid at the surface of all HD RBC when compared to LD RBC (Figure 5D), suggesting that increased adhesion to laminin of HD RBC might result from the partial loss of interaction between Lu/BCAM and GPC at the cell surface. In order to specifically address GPC sialylation levels, we used an antibody directed against sialylated forms of GPC. Flow cytometry analysis showed less GPC sialylation levels in HD than in LD RBC (Figure 5E), while no significant difference was observed in total amounts of GPC at the cell surface as determined by flow cytometry using a sialic acid-independent antiGPC antibody (Figure 5F). This result supported our hypothesis of increased HD RBC adhesion to laminin following altered cis-interactions of Lu/BCAM with GPC at the cell surface.

\section{Discussion}

Oxidative stress is an important feature of SCD and plays an important role in the pathophysiology of hemolysis, vaso-occlusion and ensuing organ damage. In this study we investigated the relationship between oxidative stress and adhesion of RBC to laminin. We report altered protein cis-interactions at the surface of sickle dense RBC that may account for the activation of RBC adhesion in the absence of signaling events and contribute to vasoocclusion. Using a microfluidic biomimetic chip, we confirm the importance of the mechanical parameter in the preferential trapping of HD RBC at a single cell level, confirming the hypothesis of the two-step model in which dense RBC contribute to the obstruction of fine blood vessels because of reduced deformability. ${ }^{13}$ In addition, we show that HD RBC adhere more firmly to laminin and are more resistant to shear stress than $L D$ RBC suggesting that they would also contribute to initiate VOCs in vivo by adhering to the vessel wall even at high shear stress. This difference is probably partly due to the increased rigidity of HD RBC, as well as to the difference in cell shape between both subtypes, with a majority of very young and round reticulocytes in the LD fraction having a smaller contact surface with the capillary wall than HD RBC that are flatter cells with a larger contact surface and a smaller section facing the flow after adhesion is initiated. This is supported by the presence of long cellular tethers and of big patches of Lu/BCAM on several adhering LD RBC indicative of important membrane dynamics in this subpopulation, which is a characteristic of reticulocytes 
known to undergo skeletal and membrane remodeling during maturation. 33,34

Several studies have investigated the dynamics and rheology of SS RBC under flow conditions using microfluidic devices. Alapan et al. assessed sickle RBC adhesion in fibronectin-coated microfluidic chips and observed significantly greater numbers of adhered non-deformable than deformable RBC. ${ }^{35}$ Our study extends these findings by comparing the adhesion of deformable and nondeformable RBC within both the young and mature populations. As a matter of fact, fibronectin is the substrate of integrin $\alpha 4 \beta 1$ that is expressed only in very young reticulocytes, restricting the analysis to a very small subpopulation of $\mathrm{RBC}$, while the laminin receptor $\mathrm{Lu} / \mathrm{BCAM}$ is expressed on $\mathrm{RBC}$ at all the maturation stages.

We show that oxidation can activate RBC adhesion to laminin by inducing post-translational modifications of Lu/BCAM that modify its distribution at the cell surface generating aggregates with high binding potential to laminin. This mechanism targets and abolishes Lu/BCAM cis-interaction with GPC at the cell surface ${ }^{32}$ and seems to be specific for Lu/BCAM and to target GPC primarily, as another IgSF member, i.e., LW/ICAM-4, was not impacted by in vitro oxidation and did not show altered distribution at the surface of SS RBCs. Abnormal RBC adhesion to laminin was reported in several pathologies ${ }^{15}$ with two triggering mechanisms including Lu/BCAM phosphorylation $^{17,24,27}$ and Lu/BCAM dissociation from the spectrinbased skeleton. ${ }^{25,26,36}$ Here, the oxidation-driven mechanism seems to be at the origin of increased adhesion of $\mathrm{HD} \mathrm{RBC}$ in the absence of Lu/BCAM phosphorylation and seems in line with the high adhesion levels of $\mathrm{HD}$ $\mathrm{RBC}$ in the absence of responsiveness to cAMP inducers. ${ }^{28}$ Moreover, although abnormal actin oxidation has been reported in irreversibly sickled cells affecting cytoskeletal dynamics, ${ }^{37}$ in our study oxidation and the subsequent loss of interaction with GPC do not alter Lu/BCAM binding to the skeleton as we did not see a difference in Lu/BCAM Triton extractability between in vitro oxidized and non-oxidized AA RBC (data not shown). This is supported by the unchanged mobility of Lu/BCAM at the surface of neuraminidase-treated RBC as measured by fluorescence recovery after photobleaching assay. ${ }^{32}$ As a matter of fact, oxidation may impact the interactions of membrane proteins with the skeleton as it was reported for Band $3^{38-41}$ but here Lu/BCAM activation seems to be triggered by modifications at the extracellular rather than the intracellular side.

During erythrocyte lifespan, sialic acid levels gradually decrease. ${ }^{42-44}$ The sialic acid levels were lower on HD than on LD RBC for all blood samples, corroborating the finding that sialoglycoproteins are enriched in membranes of young reticulocytes ${ }^{45}$ and indicating an accelerated aginglike phenotype between the two stages despite the very short lifespan of RBC in SCD. ${ }^{46,47}$ This suggests increased damage of the RBC surface in SCD, targeting the glycocalyx, probably mediated by high levels of oxidative stress effectors in the plasma including free hemoglobin. ${ }^{48}$

Our study extends our recent findings on the novel mechanism activating Lu/BCAM-mediated RBC adhesion and suggests that this mechanism could be triggered by oxidative stress activating the adhesion of dense sickle $\mathrm{RBC}$ in the absence of Lu/BCAM phosphorylation. It would be interesting to determine the impact of anti-oxidant drugs on this specific mechanism and to evaluate their potential of reducing or attenuating $\mathrm{RBC}$ adhesion in SCD.

\section{Disclosures}

No conflicts of interests to disclose.

\section{Contributions}

MALI and SDL conducted experiments, acquired and analyzed data and wrote the manuscript; VB provided blood samples and discussed data; SC, SEH, AF, MD and SA conducted experiments, analyzed data and edited the manuscript; CLVK and FRL discussed data and edited the manuscript; OF, BLP, TK and $R v B$ conducted experiments, discussed data and edited the manuscript; WEN designed research, analyzed data and wrote the manuscript.

\section{Acknowledgments}

We thank Mr Mickaël Marin, Mr Harvey Nagy and Dr Jean-Philippe Semblat for technical support.

\section{Funding}

The work was supported by the Institut National de la Sante et de la Recherche Médicale (INSERM), the Institut National de la Transfusion Sanguine, the Laboratory of Excellence GR-Ex, reference $A N R-11-L A B X-0051$, and the Laboratory of Excellence LaSIPS (ANR-10-LABX-0040-Lasips). The labex $G R-E x$ is funded by the IdEx program "Investissements d'avenir" of the French National Research Agency, reference ANR-18-IDEX-0001. MALI and SEH were funded by the Ministère de l'Enseignement Supérieur et de la Recherche (Ecole Doctorale BioSPC); they received financial support from: Club $d u$ Globule Rouge et $d u$ Fer and Société Française d'Hématologie.

\section{References}

1. Pauling L, Itano HA, Singer SJ, Wells IC. Sickle cell anemia, a molecular disease. Science. 1949;110(2865):543-548.

2. Piel FB, Steinberg MH, Rees DC. Sickle cell disease. N Engl J Med. 2017;376(16):15611573.

3. Ware RE, Montalembert MD, Tshilolo L, Abboud MR. Sickle cell disease. Lancet. 2017;6736(17):1-13.

4. Barabino GA, Platt MO, Kaul DK. Sickle cell biomechanics. Annu Rev Biomed Eng. 2010;12:345-367

5. Connes P, Lamarre $Y$, Waltz X, et al. Haemolysis and abnormal haemorheology in sickle cell anaemia. Br J Haematol. 2014;
165(4):564-572

6. Stuart MJ, Nagel RL. Sickle cell disease. Lancet. 2004;364(9442):1343-1360.

7. Hebbel RP. Beyond hemoglobin polymerization: the red blood cell membrane and sickle disease pathophysiology. Blood. 1991;77(2):214-237

8. Hebbel RP. Adhesive interactions of sickle erythrocytes with endothelium. J Clin Invest. 1997;99(11):2561-2564

9. Hebbel RP, Yamada O, Moldow CF, Jacob HS, White JG, Eaton JW. Abnormal adherence of sickle erythrocytes to cultured vascular endothelium. Possible mechanism for microvascular occlusion in sickle cell disease. J Clin Invest. 1980;65(1):154-160.

10. Cartron J, Elion J. Erythroid adhesion mole- cules in sickle cell disease: effect of hydroxyurea. Transfus Clin Biol. 2008;15(1-2):39 50

11. Kaul DK, Fabry ME. In vivo studies of sickle red blood cells. Microcirculation. 2004 11(2):153-165.

12. Kaul DK, Fabry ME, Nagel RL. Microvascular sites and characteristics of sickle cell adhesion to vascular endothelium in shear flow conditions: pathophysiological implications. Proc Natl Acad Sci U S A. 1989;86(9):3356-3360.

13. Kaul DK, Finnegan E, Barabino Ga. Sickle red cell-endothelium interactions. Microcirculation. 2009;16(1):97-111.

14. Kaul DK, Nagel RL. Sickle cell vasoocclusion: many issues and some answers. 
Experientia. 1993:49(1):5-15

15. Colin Y, Le van Kim C, El Nemer W. Red cell adhesion in human diseases. Curr Opin Hematol. 2014;21(3):186-192.

16. El Nemer W, Gane P, Colin Y, et al. The Lutheran blood group glycoproteins, the erythroid receptors for laminin, are adhesion molecules. J Biol Chem. 1998; 273(27):16686-16693.

17. Gauthier E, Wautier MP, Nemer WE, et al. Protein kinase a-dependent phosphorylation of lutheran/basal cell adhesion molecule glycoprotein regulates cell adhesion to laminin $\alpha 5$. J Biol Chem. 2005; 280(34): 30055-30062.

18. Telen MJ. Sickle cell anemia role of adhesion molecules and vascular endothelium in the pathogenesis of sickle cell disease. Hematology Am Soc Hematol Educ Program. 2007;84-90.

19. Udani M, Zen $Q$, Cottman M, et al. Basal cell adhesion molecule/lutheran protein: the receptor critical for sickle cell adhesion to laminin. J Clin Invest. 1998; 101(11):2550-2558

20.Zennadi R, Hines PC, De Castro LM, Cartron JP, Parise LV, Telen MJ. Epinephrine acts through erythroid signaling pathways to activate sickle cell adhesion to endothelium via LW-alphavbeta3 interactions. Blood. 2004:104(12):3774-3781.

21.Zennadi R, Moeller BJ, Whalen EJ, et al. Epinephrine-induced activation of LWmediated sickle cell adhesion and vasoocclusion in vivo. Blood. 2007;110(7):27082717.

22. Parsons SF, Mallinson G, Holmest $\mathrm{CH}$, et al. The Lutheran blood group glycoprotein, another member of the immunoglobulin superfamily, is widely expressed in human tissues and is developmentally regulated in human liver. Blood. 1995;92(12):5496-500.

23. Rahuel BC, Kim CLV, Mattei MG, Cartron JP, Colin Y. Unique gene encodes spliceoforms of the B-cell adhesion molecule cell surface glycoprotein of epithelial cancer and of the Lutheran blood group glycoprotein. Blood. 1996;88(5):1865-1872.

24. De Grandis MD, Cambot M, Wautier M-p, Cassinat B, Chomienne C, Colin Y, et al. JAK2V617F activates Lu/BCAM-mediated red cell adhesion in polycythemia vera through an EpoR-independent Rap1/Akt pathway. Blood. 2007;121(4):658-66.

25. An X, Gauthier E, Zhang X, et al. Adhesive activity of Lu glycoproteins is regulated by interaction with spectrin. Blood. 2008; 112(13):5212-5219.
26. Gauthier E, El W, Wautier MP, et al. Role of the interaction between Lu/BCAM and the spectrin-based membrane skeleton in the increased adhesion of hereditary spherocytosis red cells to laminin. Br J Haematol. 2009;148(3):456-465.

27. Bartolucci P, Chaar V, Picot J, et al. Decreased sickle red blood cell adhesion to laminin by hydroxyurea is associated with inhibition of Lu/BCAM protein phosphorylation. Blood. 2010;116(12):1-4

28. Hines PC, Zen Q, Burney SN, et al. Novel epinephrine and cyclic AMP-mediated activation of BCAM/Lu-dependent sickle (SS) RBC adhesion. Blood. 2003;101(8):32813287

29. McDonald JC, Whitesides GM. Poly (dimethylsiloxane) as a material for fabricating microfluidic devices. Acc Chem Res. 2002;35(7):491-499.

30. Schneider CA, Rasband WS, Eliceiri KW, Instrumentation C. NIH image to imageJ: 25 years of image analysis. Nat Methods. 2012;9(7):671-675.

31. Picot J, Ndour PA, Lefevre SD, et al. A biomimetic microfluidic chip to study the circulation and mechanical retention of red blood cells in the spleen. Am J Hematol. 2015;90(4):339-345.

32. Klei TRL, Back DZD, Asif PJ, et al. Glycophorin-C sialylation regulates Lu/BCAM adhesive capacity during erythrocyte aging. Blood. 2018;2(1):14-24.

33. Chasis JA, Prenant M, Leung A, Mohandas $\mathrm{N}$. Membrane assembly and remodeling during reticulocyte maturation. Blood. 1989;74(3):1112-1120.

34. Mohandas N, Groner W. Cell membrane and volume changes during red cell development and aging. Ann N Y Acad Sci. 1989;554:217-224.

35. Alapan Y, Matsuyama Y, Little JA, Gurkan UA. Dynamic deformability of sickle red blood cells in microphysiological flow. Technology (Singap World Sci). 2016; 4(2):71-79.

36. Wandersee NJ, Olson SC, Holzhauer SL, Hoffmann RG, Barker JE, Hillery CA. Increased erythrocyte adhesion in mice and humans with hereditary spherocytosis and hereditary elliptocytosis. Blood. 2004; 103(2):710-717

37. Shartava A, Monteiro CA, Bencsath FA, et al. A posttranslational modification of betaactin contributes to the slow dissociation of the spectrin-protein 4.1-actin complex of irreversibly sickled cells. J Cell Biol. 1995; 128(5):805-818.
38. Arashiki N, Kimata N, Manno S, Mohandas N, Takakuwa Y. Membrane peroxidation and methemoglobin formation are both necessary for Band 3 clustering: mechanistic insights into human erythrocyte senescence. Biochemistry. 2013;52(34):5760 5769 .

39. Mannu F, Arese P, Cappellini MD, et al Role of hemichrome binding to erythrocyte membrane in the generation of Band-3 alterations in B-Thalassemia intermedia erythtrocytes. Blood. 1995;86(5):2014 2020

40. Pantaleo A, Giribaldi G, Mannu F, Arese P, Turrini F. Naturally occurring anti-band 3 antibodies and red blood cell removal under physiological and pathological conditions. Autoimm Rev. 2008;7(6):457-462.

41. Noomuna P, Risinger M, Zhou S, et al. Inhibition of Band 3 tyrosine phosphorylation: a new mechanism for treatment of sickle cell disease. Br J Haematol. 2020;190 (4):599-609.

42. Hadengue AL, Del-pino M, Simon A, Levenson J. Erythrocyte disaggregation shear stress, sialic acid, and cell aging in humans. Hypertension. 1998;32(2):324 330.

43. Huang Y-x, Tuo W-w, Wang D, Kang L-1, Chen X-y, Luo M. Restoring the youth of aged red blood cells and extending their lifespan in circulation by remodelling membrane sialic acid. J Cell Mol Med. 2016;20 (2):294-301

44. Shinozuka T. Changes in human red blood cells during aging in vivo. Kelo J Med. 1994; 43(3):155-163.

45. Skutelsky E, Farquhar M. Variations in distribution of Con A receptor sites and anionic groups during red blood cell differentiation in the rat. J Cell Biol. 1976;71(1):218231.

46. Franco RS, Lohmann J, Silberstein EB, Mayfield-pratt G, Palascak M, Nemeth TA. Time-dependent changes in the density and hemoglobin $\mathrm{F}$ content of biotin-labeled sickle cells. J Clin Invest. 1998;101(12): 2730-2740

47. Quinn CT, Smith EP, Arbabi S, et al. Biochemical surrogate markers of hemolysis do not correlate with directly measured erythrocyte survival in sickle cell anemia. Am J Hematol. 2016;91(12):1195-1201.

48. Rifkind JM, Mohanty JG, Nagababu E. The pathophysiology of extracellular hemoglobin associated with enhanced oxidative reactions. Front Physiol. 2015;5:500. 